\title{
HST/NICMOS2 coronagraphic observations of the circumstellar environment of three old PMS stars: HD 100546, SAO 206462 and MWC 480*
}

\author{
J. C. Augereau ${ }^{1}$, A. M. Lagrange ${ }^{1}$, D. Mouillet $^{1}$, and F. Ménard ${ }^{1,2}$ \\ 1 Laboratoire d'Astrophysique de l'Observatoire de Grenoble, Université J. Fourier, CNRS, BP 53, \\ 38041 Grenoble Cedex 9, France \\ 2 Canada-France-Hawaii Telescope Corporation, PO Box 1597, Kamuela, HI 96743, USA
}

Received 16 August 2000 / Accepted 29 September 2000

\begin{abstract}
The close environment of four old Pre-Main Sequence stars has been observed thanks to the coronagraphic mode of the HST/NICMOS2 camera at $\lambda=1.6 \mu \mathrm{m}$. In the course of this program, a circumstellar annulus around HD 141569 was detected and has already been presented in Augereau et al. (1999b). In this paper, we report the detection of an elliptical structure around the Herbig Be star HD 100546 extending from the very close edge of the coronagraphic mask $(\sim 50 \mathrm{AU})$ to $350-380 \mathrm{AU}\left(3.5-3.8^{\prime \prime}\right)$ from the star. The axis ratio gives a disk inclination of $51^{\circ} \pm 3^{\circ}$ to the line-of-sight and a position angle of $161^{\circ} \pm 5^{\circ}$, measured east of north. At $50 \mathrm{AU}$, the disk has a surface brightness between 10.5 and $11 \mathrm{mag} / \operatorname{arcsec}^{2}$, then follows a $-2.92 \pm 0.04$ radial power law up to $250-270 \mathrm{AU}$ and finally falls as $r^{-5.5 \pm 0.2}$. The inferred optical thickness suggests that the disk is at least marginally optically thick inside $80 \mathrm{AU}$ and optically thin further out. Combined with anisotropic scattering properties, this could explain the shape of a brightness asymmetry observed along the minor axis of the disk. This asymmetry needs to be confirmed. The circumstellar disks around SAO 206462 and MWC 480 are not resolved, leading to constraints on the dust distribution. A tight binary system separated by only $0.32^{\prime \prime} \pm 0.04^{\prime \prime}$ is nevertheless detected in the close vicinity of SAO 206462.
\end{abstract}

Key words. stars: circumstellar matter - stars: HD 100546, SAO 206462, MWC 480 - stars: pre-main sequence

\section{Introduction}

Dust is present around a large fraction of Main Sequence (MS) stars, as shown by IRAS (Aumann et al. 1984). Moreover, spectroscopic (e.g. Queloz et al. 2000) and photometric (Charbonneau et al. 2000) detections indicate that planetary systems are quite common. In some cases, the dust lifetime is so short compared to the star's age that it has to be replenished, probably through collisions between planetesimals or through evaporation (Backman $\&$ Paresce 1993). The best example so far of these second generation disks is the one surrounding $\beta$ Pictoris aged more than $20 \mathrm{Myr}$ (Barrado y Navascués et al. 1999). Recently, submillimeter images were used to resolve the dust emission around a few isolated MS stars with

Send offprint requests to: J. C. Augereau,

e-mail: augereau@obs.ujf-grenoble.fr

* Based on observations with the NASA/ESA Hubble Space Telescope, obtained at the Space Telescope Science Institute, which is operated by the Association of Universities for Research in Astronomy, Inc. under NASA contract No. NAS5-26555. different spectral types and older than 0.1 Gyr (Holland et al. 1998; Greaves et al. 1998).

Circumstellar disks are also present around young objects, as clearly demonstrated by HST (Padgett et al. 1999, for instance), and by ground-based observations (Dutrey et al. 1996). The latter detected circumstellar disks around a sample of CTTs in the Taurus cloud, but not yet around post $\mathrm{T}$ Tauri stars. This is in general agreement with Zuckerman \& Becklin (1993) and more recently Habing et al. (1999) who have shown the rapid decrease of the circumstellar dust mass as the stars evolve to the Main Sequence (see also Holland et al. 1998). Gas infall is also observed towards a few Herbig Ae/Be (hereafter HAeBe) stars (e.g. Grady et al. 1996; de Winter et al. 1999), tentatively interpreted as the result of comet evaporation. In this scheme, this could indicate that planetesimal formation in the nebula is quite common and rapid, as theoretically expected.

Very few disks have been detected around HAeBe and more evolved Pre Main-Sequence (PMS) stars (HD 163296 and MWC 480, Mannings \& Sargent 1997a; Mannings et al. 1997b). A recent study of HR 4796, 
an 8 Myr star, has evidenced that the disk is at least partly second generation and that meter-size bodies should already be present (Augereau et al. 1999a). Similar conclusions could apply for the HD 141569 disk (Augereau et al. 1999b). In both cases, the analysis relies on detailed studies of the resolved images and spectral energy distributions (SEDs). From a general point of view, the nature of transient disks, the role of replenishing planetesimals if any, and the most relevant physical processes in these environments are still to be clarified.

The general evolutionary scenario to be tested and refined is then that:

1) the protoplanetary disks around young, embedded stars disperse out before the star reaches the main sequence through dynamical activity and interaction with stellar radiation, and that;

2) new optically thin circumstellar disks may be sustained around older MS stars by destruction processes among planetesimals (collisions, evaporation), formed during the Pre-Main Sequence (PMS) phase.

Analysis of the transition between these two phases, when the original young disk is eroded, is critical to validate the above scenario. Such a study can be performed on disks around evolved PMS stars. Critical points for this study are the analysis of opacity to stellar radiation and the time-scales of collisional processes. Both of these points require detailed information on the spatial distribution and amount of circumstellar dust.

During HST/Cycle 7, we probed in coronagraphic mode the environment of four evolved PMS stars with the NICMOS2 camera. As part of this program, we reported the positive detection of an extended ( $>400 \mathrm{AU}$ in radius) structure around HD 141569 in Augereau et al. (1999b). The detection was confirmed by Weinberger et al. (1999). We address in the present paper our results for three other sources of large interest among old PMS stars: HD 100546, SAO 206462 and MWC 480. After a brief description of the observing strategy, journal of observation and reduction procedure (Sect. 2), we present in Sect. 3 the results for HD 100546. An extended structure is detected and interpreted in terms of circumstellar dust arranged within an inclined disk. Detection limits are derived from unresolved structures around SAO 206462 and MWC 480 (Sects. 4 and 5) and in each case we discuss the implications on the dust distribution. We also detail the close vicinity of SAO 206462 which exhibits a tight binary system.

\section{HST/NICMOS2 observations and data analysis}

\subsection{Target selection}

Our aim was to image the circumstellar environments of transition (old PMS) objects to establish the missing link between disks around young embedded objects and $\beta$ Pic-like stars. We compare the optically thick disks of young stars with the optically thin disk of $\beta$ Pictoris. Our four targets (HD 100546, SAO 206462, MWC 480 and HD 141569) were selected according to the following criteria:

- a PMS star close to the MS, which can be characterized by a large IRAS excess, together with an age estimation (via photometry or spectroscopy) of about $10^{6}-10^{7}$ years; - surrounded by circumstellar dust (IR excess) and CO. $\mathrm{CO}$ detection indeed provides a good indication for the gas to be extended;

- must have been observed at various wavelengths to allow comprehensive investigation.

\subsection{Observing strategy}

The high level of contrast between the investigated circumstellar environments and their central star implies the use of coronagraphic techniques. The subtraction of residual Point Spread Fonction (PSF) wings is required to take full benefit of the coronagraph's high contrast capabilities. For that, we observed in the same optical configuration a comparison star within a few degrees of the science target, of similar or slightly higher brightness, with similar spectral type and thought to be free of circumstellar matter.

Comparison stars need to be observed close in time to each corresponding science target observation because of the slight possible variation of the PSF with time. This method has proved to be efficient for ground-based coronagraphic data with adaptive optics, especially because it is still efficient if the PSF is not perfectly symmetrical. It also allows the detection of circumstellar disks far from edge-on orientation.

\subsection{NICMOS2 data}

We obtained coronagraphic images of our four targets with the HST/NICMOS2 camera between February and November, 1998. All the observations were performed in Filter F160W $\left(\lambda_{\mathrm{c}}=1.6 \mu \mathrm{m}, \Delta \lambda=0.4 \mu \mathrm{m}\right)$. For the three targets presented in this paper, the observing log is summarized in Table 1. The individual integration times were determined so as to stay below the saturation limit of the detector on the brightest pixels (near the central hole edge area). Filter F160W was selected for optimum detectability: the PSF is well sampled and the background (zodiacal light and thermal background) remains low.

\subsection{Reduction procedure, photometry and astrometry}

For each observed star, the calibrated files (in counts/s) provided by the STScI are co-added to form a single image of the star during a same orbit. The reduction procedure then consists in subtracting the comparison star carefully scaled to the star of interest. The determination of the scaling factor is critical since a small change can significantly modify the photometry or in the worst case induce artifacts. It is assessed by azimuthally averaging the profile resulting from the division between the star of interest by the reference star images (see also Augereau et al. 1999b). 
Table 1. Observing log. Note that SAO 206462 and SAO 206463 form a visual binary system (HD 135344). References: (a) Waelkens et al. (1990a,b), (b) Coulson et al. 1995, (c) Simbad (CDS), (d) Myers et al. (1998)

\begin{tabular}{|c|c|c|c|c|c|c|}
\hline & Star Name & Spectral Type & $V$ mag & Date & Integration Time & Same Orbit? \\
\hline Object: & HD 100546 & B9Vne & 6.68 & Nov. 09, 1998 & $16 \times 47.958 \mathrm{~s}=12 \mathrm{~m} 47 \mathrm{~s}$ & \\
\hline Reference: & HD 106797 & $\mathrm{~A} 0 \mathrm{~V}$ & 6.07 & Nov. 09,1998 & $16 \times 47.958 \mathrm{~s}=12 \mathrm{~m} 47 \mathrm{~s}$ & Yes \\
\hline Object: & SAO 206462 & $\mathrm{~F} 4 \mathrm{Ve}^{(a)} ; \mathrm{F} 8 \mathrm{~V}^{(b)}$ & 8.65 & Aug. 22, 1998 & $7 \times 111.931 \mathrm{~s}=13 \mathrm{~m} \mathrm{03s}$ & \\
\hline Reference: & SAO 206463 & $\mathrm{~F} 2^{(c)} ; \mathrm{A} 2^{(d)}$ & 7.9 & Aug. 22, 1998 & $7 \times 191.961 \mathrm{~s}=22 \mathrm{~m} \mathrm{24s}$ & Yes \\
\hline Object: & MWC 480 & $\mathrm{~A} 2$ & 7.72 & Feb. 24, 1998 & $11 \times 39.953 \mathrm{~s}=7 \mathrm{~m} 20 \mathrm{~s}$ & \\
\hline Reference: & HD 29646 & $\mathrm{~A} 2 \mathrm{~V}$ & 5.73 & Feb. 24, 1998 & $11 \times 31.959 \mathrm{~s}=5 \mathrm{~m} 52 \mathrm{~s}$ & Yes \\
\hline
\end{tabular}

Table 2. Some known astrophysical parameters of the target sources presented in this paper. References: ${ }^{(a)}$ van den Ancker et al. (1997), ${ }^{(b)}$ Sylvester et al. (1996), ${ }^{(c)}$ Zuckerman et al. (1995), (d) Coulson et al. (1995), (e) Dunkin et al. (1997), (f) van den ancker et al. (1998), ${ }^{(g)}$ Mannings et al. (1997b)

\begin{tabular}{ccccccc}
\hline Star Name & Distance $[\mathrm{pc}]$ & Luminosity $\left[L_{\odot}\right]$ & Mass $\left[M_{\odot}\right]$ & $T_{\text {eff }}[\mathrm{K}]$ & Age $[\mathrm{Myr}]$ & Other Name \\
\hline HD 100546 & $103_{-6}^{+7(a)}$ & $32.4_{-3.5}^{+4.5}(a)$ & $2.4 \pm 0.1^{(a)}$ & $10470^{(a)}$ & $>10^{(a)}$ & SAO 251457 \\
SAO 206462 & $84^{(b)} ; 100^{(c)}$ & & & $6250^{(d)} ; 6660^{(e)}$ & HD 135344 \\
MWC 480 & $131_{-18}^{+24(f)}$ & $32.4_{-8.4}^{+13.3(f)}$ & $2.2 \pm 0.3^{(f)} ; 2.3_{-0.3}^{+0.1}(g)$ & $8710^{(f)}$ & $2.5_{-0.9}^{+1.5}(f) ; 6^{(g)}$ & HD 31648 \\
\hline
\end{tabular}

We use the $2.07710^{-6} \mathrm{Jy} \mathrm{s} /$ counts factor to convert NICMOS count rates to absolute fluxes and a zero point flux density of $1040.7 \mathrm{Jy}$ to convert to magnitudes (NICMOS data handbook, version 4.0, dated december 1999). Point source photometry is obtained by integrating the total flux within a $0.5^{\prime \prime}$ radius circular aperture, then applying a 1.15 correcting factor to compensate for the flux which fell out of this aperture (NICMOS photometry update web page).

The pixel scale slowly varies with time but stays within the range $0.075^{\prime \prime}-0.076^{\prime \prime}$ in average.

\section{HD 100546}

\subsection{A Herbig Be star close to the ZAMS}

The HD 100546 star has been intensively studied since it was identified as a member of the HAeBe group $(\mathrm{Hu}$ et al. 1989). Indeed, HD 100546 is a B9Vne star showing a strong infrared excess peaked at about $25 \mu \mathrm{m}$ (IRAS) due to circumstellar material and then fulfills the criterions proposed by Waters \& Waelkens (1998) to identify HAeBe stars. Some astrophysical parameters are summarized in Table 2.

The position of the star in the HR diagram indicates that HD 100546 is close to the ZAMS leading to an estimated age larger than $10 \mathrm{Myr}$ (van den Ancker et al. 1997). This star, associated with the dark cloud DC 296.27.9 (Hu et al. 1989; Vieira et al. 1999), is surrounded by a large amount of dust: between a few tens (Bouwman et al. 2000) and a few hundred Earth masses (Henning et al. 1998). The dust is rich in $\mathrm{C}$ and $\mathrm{O}$ and in particular PAH and silicates (Malfait et al. 1998). The latter are very valuable since they constrain the dust optical properties. ISO observations also evidenced similarities between the
$10 \mu \mathrm{m}$ emission of the circumstellar dust and that of comet Hale-Bopp (Crovisier et al.1997; Malfait et al. 1998).

As commonly observed for other HAeBe stars, HD 100546 exhibits photometric, polarization and spectroscopic variability (Grady et al. 1996, 1997; van den Ancker et al. 1998; Yudin \& Evans 1998; Clarke et al. 1999; Vieira et al. 1999). The interpretation of such events in particular in terms of star-grazing comets (Grady et al. 1997; Vieira et al. 1999) remains however uncertain (Lagrange et al. 2000; Beust et al. 2000).

Another issue concerns the very distribution of the material surrounding HD 100546. The disk detection in scattered light between $40\left(0.4^{\prime \prime}\right)$ and $200 \mathrm{AU}\left(2^{\prime \prime}\right)$ by Pantin et al. (2000) reveals $\mathrm{a} \sim 50^{\circ}$ inclined disk almost in the SE-NW direction. At $\lambda=1.3 \mathrm{~mm}$, an extension as large as $15-20^{\prime \prime}$ in radius and close to the detection limit is also reported by Henning et al. (1998) in the same direction. From the theoretical point of view, it is unclear whether the circumstellar material arranges within a single disk. For instance, the presence of an additional envelope is proposed to reproduce the full spectral energy distribution (Henning et al. 1994) and to explain specific spectroscopic events (Vieira et al. 1999).

\subsection{Results}

\subsubsection{Resolved disk and vicinity}

The azimuthally averaged radial profile of the ratio between HD 100546 and the reference star HD 106797 shows two basic distinct regimes: up to $3.5-3.8^{\prime \prime}$, the profile decreases with distance from the star then reaches a plateau further out. The same behavior arises using the A2 and A1 reference stars dedicated to MWC 480 and HD 141569 respectively. A resolved structure around HD 100546 is then detected below $3.5-3.8^{\prime \prime}$. A $3 \sigma$ uncertainty of $2 \%$ 


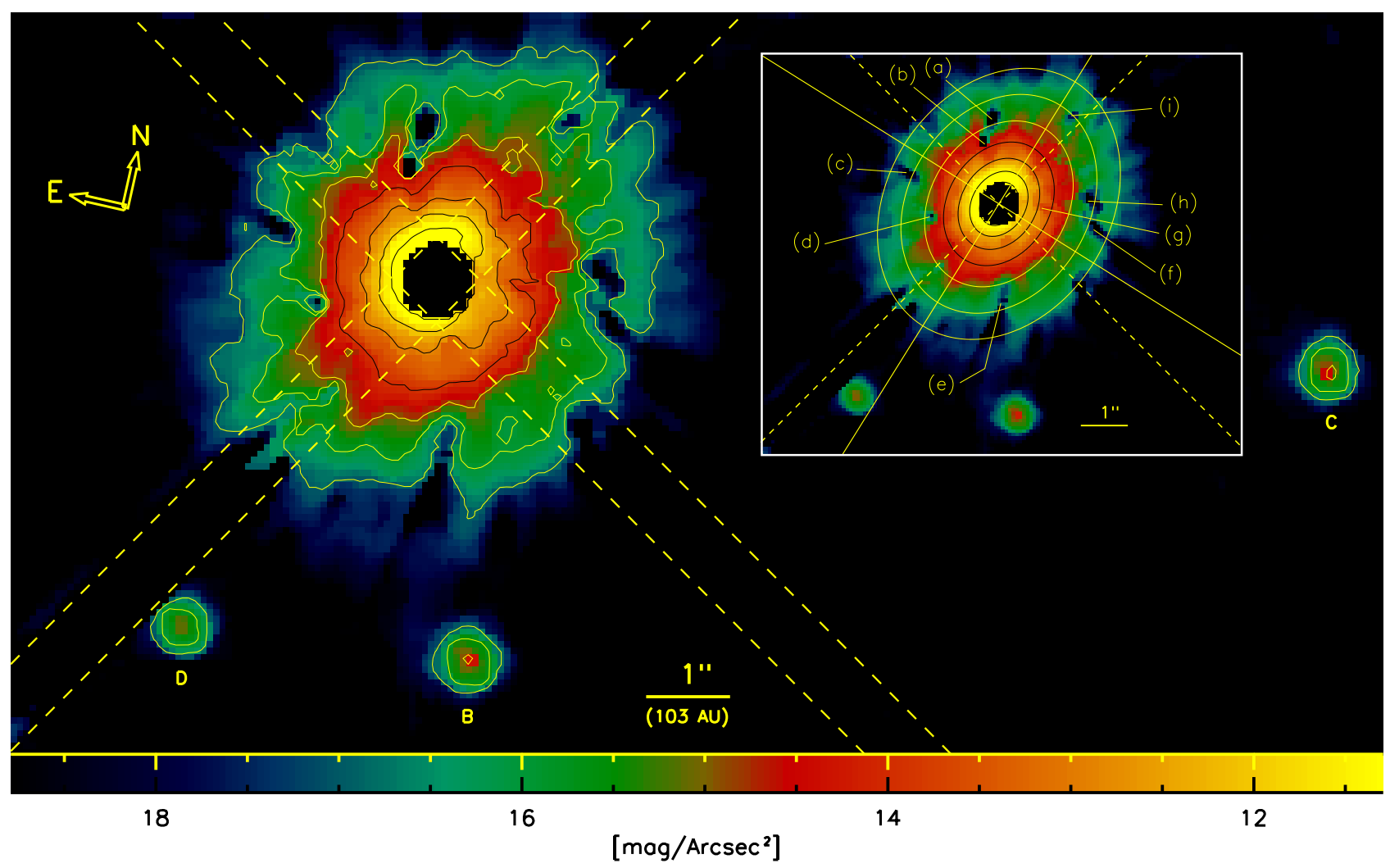

Fig. 1. Reduced image of the HD 100546 disk observed in coronagraphic mode at $1.6 \mu$ mith the HST/NICMOS2 camera. The coronagraphic mask is $0.7^{\prime \prime}$ in diameter $\left(\sim 9\right.$ pixels) whereas the numerical mask in the figure is $0.9^{\prime \prime}$. Isophotes from $11 \mathrm{mag} / \operatorname{arcsec}^{2}$ to $17 \mathrm{mag} / \operatorname{arcsec}^{2}$ are indicated. The dashed lines give the width and the position of the spider arms. The small panel in the upper right corner shows the result of isophotes ellipse fitting. Inhomogeneities due to the detector or to secondary spider diffraction spikes (which produce some kinds of fingers) are responsible for black spots. The most noticeable are labeled (a) to $(i)$. In the same small panel, the plain lines represent the major and minor axis of the observed structure and the dashed ones represent the position of the spider arms

is obtained on the scaling factor used to subtract the reference star to HD 100546 which directly impacts on the precision of photometric measurements.

The final calibrated image is shown in Fig. 1 and reveals an extended elliptical structure centered on the star. We interpret this structure as circumstellar material located in an inclined disk. Visual inspection suggests the direction subtended by the spider arm at a position angle (PA, measured east of north) of $\sim 148^{\circ}$ as an axis of symmetry for the inclined disk, at least at large distances. A more robust method (ellipse-fitting to the isophotes) leads to a $\mathrm{PA}$ of $161^{\circ} \pm 5^{\circ}$ but the result might be affected by different noise sources. Whatever the method, the global direction of the disk is consistent with the observations of Pantin et al. (2000) but the precise PA is found to be at least $20^{\circ}$ larger than their measurements. The axis ratio corresponds to a disk inclination of $51^{\circ} \pm 3^{\circ}$ with respect to the line of sight, assuming that the disk is axisymmetric, and agrees with the results of Pantin et al. (2000). We measure a total flux density of $73 \pm 7 \mathrm{mJy}$ (the uncertainty is dominated by uncertainty in the scaling factor used to subtract the PSF star).
Table 3. Projected distances, position angles (PAs) and magnitudes in filter F160W $\left(\lambda_{\mathrm{c}} \simeq 1.6 \mu \mathrm{m}\right)$ of the 3 brightest visual companion stars detected in the vicinity of HD 100546. The labels refer to Fig. 1. $3 \sigma$ uncertainties in the positions and PAs are mainly due to the uncertainty in the central star position below the mask but also to the uncertainty in the companion star centroid. The $3 \sigma$ photometric uncertainty is induced by the uncertainty on the scaling factor used to subtract the PSF star to the star of interest (Sect. 2.4)

\begin{tabular}{cccc}
\hline Label & $\begin{array}{c}\text { Projected distance } \\
{[\text { Arcsec] }}\end{array}$ & $\begin{array}{c}\text { PA } \\
{[\text { degrees }]}\end{array}$ & $\begin{array}{c}\text { magnitude } \\
\text { filter F160W }\end{array}$ \\
\hline $\mathrm{B}$ & $4.50 \pm 0.15$ & $197.7 \pm 1.1$ & $15.74 \pm 0.04$ \\
$\mathrm{C}$ & $10.80 \pm 0.15$ & $277.1 \pm 0.5$ & $15.79 \pm 0.11$ \\
$\mathrm{D}$ & $5.09 \pm 0.15$ & $156.3 \pm 0.9$ & $16.09 \pm 0.08$ \\
\hline
\end{tabular}

Some companion stars are also detected in the close vicinity of HD 100546. The three brightest ones are shown in Fig. 1 and their measured astronomical parameters (projected separations, PAs and magnitudes) are summarized in Table 3. 

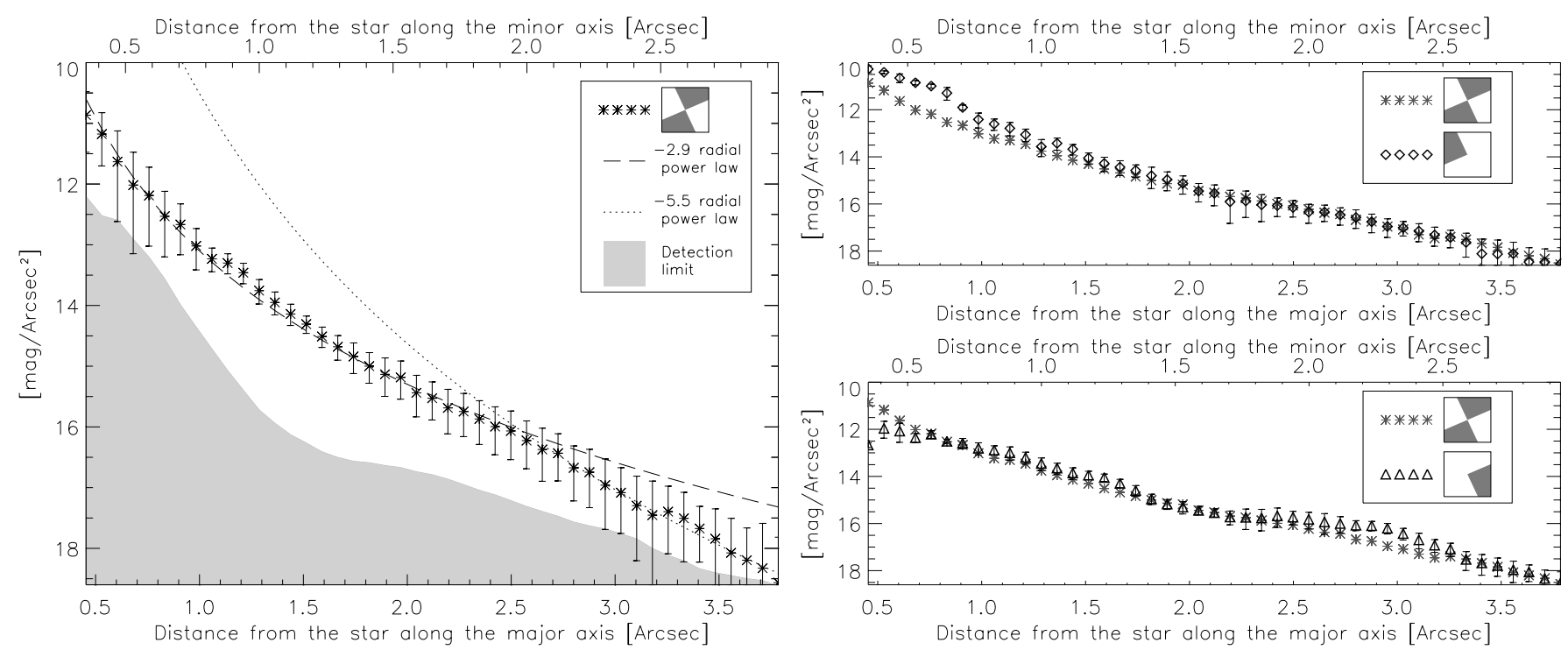

Fig. 2. The HD 100546 disk radial surface brightness profiles azimuthally averaged on elliptical contours assuming PA $=161^{\circ}$ for the major axis and an eccentricity of $e=0.63$. The left panel shows the major axis profile and the right ones the minor axis profiles. The dark grey areas in the small boxes in the legend mimic the angular sectors centered on the star used in each case. These results are discussed in Sects. 3.2.2 and 3.2.3

\subsubsection{Radial surface brightness profiles}

The radial brightness profiles of the disk are assessed by azimuthally averaging the surface brightness on elliptical contours. The radial profile measured towards the major axis of the disk is shown in the left panel of Fig. 2. It reveals a smooth and continuous decrease of the surface brightness with the distance from the star from the very close edge of the coronagraphic mask up to $2.5^{\prime \prime}$. Beyond $2.5^{\prime \prime}$, the profile is steeper and reaches the detection limit (grey area in Fig. 2) at 3.5-3.8" . Basic radial power-laws properly fit this measured surface brightness profile with indexes $-2.92 \pm 0.04$ in the radial range $\left[0.5^{\prime \prime}, 2.5^{\prime \prime}\right]$ and $-5.5 \pm 0.2$ outside of $2.7^{\prime \prime}$.

The surface brightness profiles along the minor axis (NE and SW sides of the disk) are shown in the right panels of Fig. 2. To allow direct comparison with the results along the major axis, we plot the profiles versus deprojected distances. They correspond to the observed distances along the minor axis over $\sin \left(51^{\circ}\right)$. As a confirmation of the isophote ellipse fitting procedure, the main shape of the minor axis profiles superimposes well on the major axis one.

\subsubsection{NE-SW brightness asymmetry}

However, a significant brightness enhancement arises in the NE direction mainly below $0.7-0.8^{\prime \prime}$ in projected distances but not in the opposite direction. Also in the same radial range, the behavior of the SW profile better follows the major axis profile down to $\sim 0.5^{\prime \prime}$. Below this distance, the SW side of the disk is slightly fainter but with a poor level of confidence (very close to the detection limit). This NE-SW brightness asymmetry is clearly evidenced in Fig. 1.
To emphasize the asymmetry, we subtract a synthetic disk which fits the disk isophotes to the observed disk of HD 100546. In the NE side, the subtraction shown in Fig. 3 reveals an excess of flux up to $1.1 \mathrm{mag} / \operatorname{arcsec}^{2}$ at $0.5^{\prime \prime}-0.6^{\prime \prime}$ collimated in a direction close to the minor axis of the disk (plain line). In the SW side, the systematic pattern at about $2.2^{\prime \prime}$, responsible for the shape of the radial profile shown in the bottom right panel of Fig. 2, is clearly seen.

We first checked whether this asymmetry could result from a bad centering of the PSF star during the reduction procedure. To significantly decrease the inner brightness asymmetry, the reference star has to be shifted by more than 1.5 pixel in the NE direction. This leads to unrealistic strong asymmetries in the rest of the image which characterize an obvious bad centering of the subtracted PSF star. The asymmetry arises close to the position of a diffraction spike which might seem suspicious. However, due to a good alignment, the diffraction spikes were completely removed during PSF subtraction and did not require any further processing which might directly cause the effect.

Could the effect then be due to instrumental effects? HD 100546 and the reference star have been observed during the same orbit. Therefore, orbit-to-orbit variations, such as the migration $(\sim 0.25$ pixel $)$ of the coronagraphic hole on the detector or defocus (Schultz et al. 1999) cannot account for the observed effect. Another issue concerns the mis-centering of the star behind the mask during target acquisition. An accuracy of a few tenths of pixel is reached when centering PSF on the occulting mask but a mis-centering of the star by only a third of pixel can produce brightness asymmetries mainly within a radius of $0.4^{\prime \prime}-0.5^{\prime \prime}$ (Schultz et al. 1999). Observations at different 

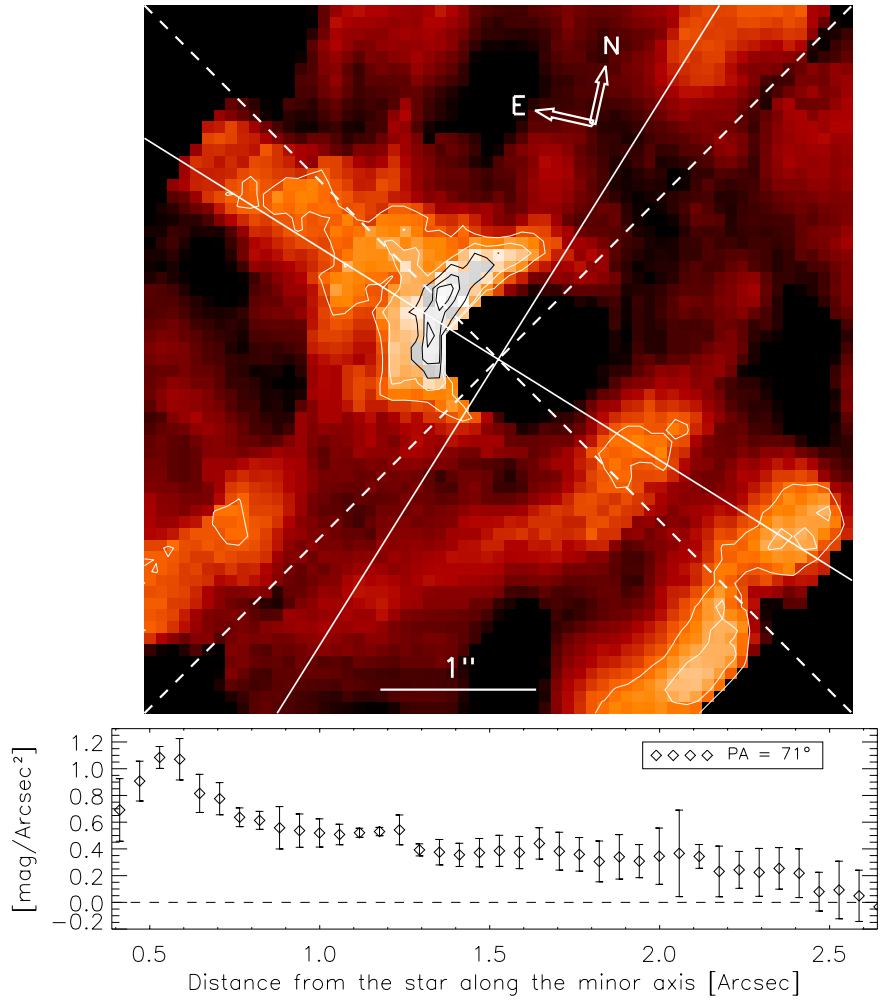

Fig. 3. Upper panel: Residue resulting from the subtraction of a synthetic disk with an axisymmetrical surface brightness strictly identical to the one shown in the left panel of Fig. 2 and inclined at $51^{\circ}$ from edge-on towards $\mathrm{PA}=161^{\circ}$ to the reduced image shown in Fig. 1. For each pattern, the lowest level is $0.4 \mathrm{mag} / \operatorname{arcsec}^{2}$. The white contours are spaced at 0.4 and $0.6 \mathrm{mag} / \operatorname{arcsec}^{2}$, the black ones at $0.8,1$ and $1.1 \mathrm{mag} / \operatorname{arcsec}^{2}$. As for Fig. 1, the plain lines represent the major and minor axis and the dashed ones the position of spider arms. Lower panel: radial profile along the NE minor axis averaged for each distance from the star over 9 pixels in the perpendicular direction. The subtraction highlights the NE-SW brightness asymmetry. It allows to quantify this asymmetry and to determine the PA of the excess

roll angles would help to check whether the asymmetry is real or an artifact.

\subsection{Discussion and interpretation}

\subsubsection{Disk radiation to stellar luminosity ratio at $1.6 \mu \mathrm{m}$}

The measured flux density of the disk at $1.6 \mu \mathrm{m}$ corresponds to $10.4 \pm 0.1 \mathrm{mag}$ according to the zero point flux provided by the STScI. On the other hand, the $V-H$ color index observed by Hu et al. (1989) and more recently Kurucz spectrum fitting by Malfait et al. (1998) indicate that the disk emission represents $55 \%$ to $60 \%$ of the total flux in the $H$-band. This excess may be due to the thermal emission of hot grains at very short distances from the central star, presumably close to the grain sublimation limit (a few fraction of AU, see also Fig. 6). Obviously

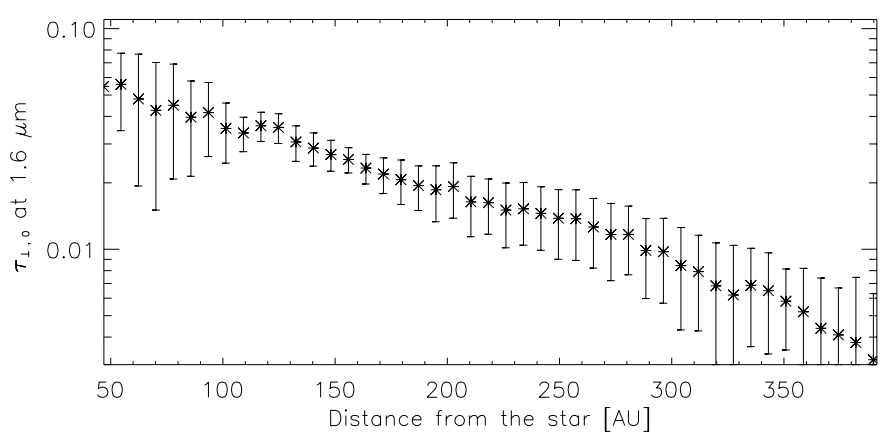

Fig. 4. $1.6 \mu \mathrm{m}$ normal optical depth derived from the observed radial surface brightness distribution of the HD 100546 disk in the optically thin approach. Between 50 and $100 \mathrm{AU}$, it is a factor of between 40 and 50 less than the normal optical thickness inferred by Pantin et al. (2000) at $1.25 \mu \mathrm{m}$ ( $J$ band)

this excess falls below the coronagraphic mask used during present observations (the mask radius is $0.35^{\prime \prime}$ or $\sim 36 \mathrm{AU}$ in situ according to Hipparcos measurements). Assuming $H=5.88$ for HD 100546 (Malfait et al. 1998), our flux density measurement provides a lower limit of $410^{-2}$ for the scattered to photospheric flux ratio at $1.6 \mu \mathrm{m}$ with an uncertainty of $15 \%$. This is about 2.5 less than the ratio inferred by Pantin et al. (2000) in $J$ band.

\subsubsection{Normal optical thickness of the disk}

As a first step in analyzing the data, we assume that the disk is optically thin whatever the direction. Since the disk is inclined with respect to the line-of-sight, it is then straightforward to derive the normal optical thickness $\tau_{\perp}(r)$ at $1.6 \mu \mathrm{m}$. This requires some reasonable assumptions of the optical dust properties. Both the method and its assumptions are summarized in Appendix A. The result is shown in the upper panel of Fig. 4. In this simple approach, the disk appears indeed optically thin vertically at $1.6 \mu \mathrm{m}$ everywhere in the resolved radial range but $\tau_{\perp}$ reaches a quite high maximum of $\sim 0.06$ close to the mask's edge.

Following Krist et al. (2000), we then assess the midplane optical depth by assuming that the vertical shape of the disk is a Gaussian with a similar scale height of $20 \mathrm{AU}$ at $140 \mathrm{AU}$ from the star and a $\beta$ disk flaring index of 1 . We find a 0.15 midplane optical depth between $50 \mathrm{AU}$ and the outer edge of the resolved disk. It falls below 0.1 between $\sim 80 \mathrm{AU}$ and the outer edge. This would indicate that the optically thin regime may not apply in the inner regions of the disk and that part of the stellar light is obscured at large distances in the midplane leading to an underestimation of $\tau_{\perp}$. This is in line with the large millimeter flux (Henning et al. 1994, 1998), that suggests that the disk is at least marginally optically thick in part. 


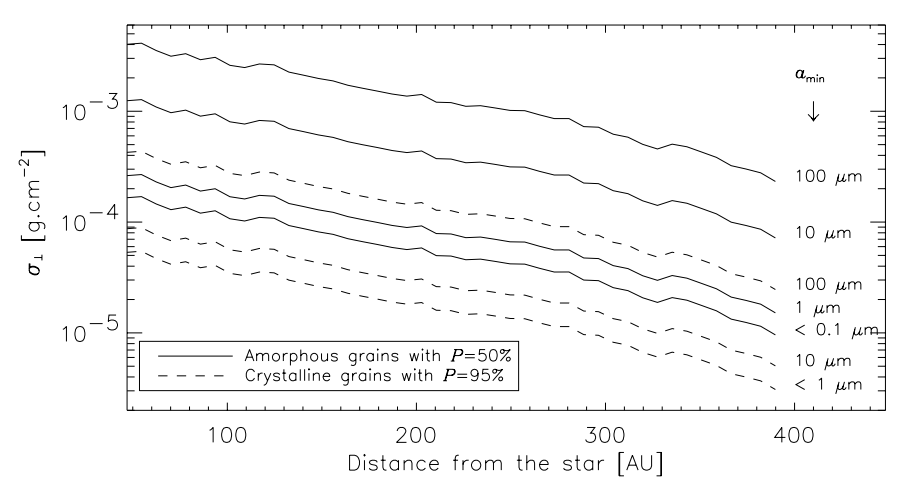

Fig. 5. Normal surface densities of the disk which reproduce, in the optically thin approach, the scattered light observations for different minimum grain sizes and the two types of grains considered. Note that surface densities as well as masses should be scaled by a factor $\sqrt{a_{\max }[\mathrm{cm}]}$ if the maximum grain size is not $1 \mathrm{~cm}$, as is assumed here

\subsubsection{Normal surface density and minimum mass of the dust disk}

The optically thin approach is useful to determine a lower limit on the normal surface density $\sigma_{\perp}(r)$ in solids (see Appendix B). Since we assume a grain size distribution proportional to $a^{-3.5}, \sigma_{\perp}(r)$ depends both on the minimum $\left(a_{\min }\right)$ and maximum $\left(a_{\max }\right)$ grain sizes in the disk. Dust grains are assumed to be icy porous aggregates made of a silicate core and coated by organic refractories. Two dust chemical compositions are considered: amorphous and crystalline grains with porosities of 0.5 and 0.95 respectively (see Appendix A and Augereau et al. 1999a, for more details on the model used). $\sigma_{\perp}(r)$ is plotted in Fig. 5 for different minimum grain sizes. Between $50 \mathrm{AU}$ and $100 \mathrm{AU}$ for instance, $\sigma_{\perp}(r)$ is as large as a few $10^{-5} \mathrm{~g} \mathrm{~cm}^{-2}$ and about one order of magnitude larger if grains are at the blow-out size limit $(\sim 13 \mu \mathrm{m}$, see Fig. A.1). In terms of dust mass between $50 \mathrm{AU}$ and $390 \mathrm{AU}$, this corresponds to a few Earth masses at most (the dust mass is about $\left.3750 \times \sigma_{\perp}(50 \mathrm{AU}) M_{\oplus}\right)$ and is at least one order of magnitude less than dust masses inferred from infrared or millimeter excesses (Bouwman et al. 2000; Henning et al. 1998). Obviously, if meter-sized bodies are formed, the normal surface density as well as the mass in solids should be multiplied by at least a factor of 10 . The dust mass may also be significantly larger if the inferred optical thickness is underestimated.

\subsubsection{Shape of the dust distribution in the optically thin approach}

$\sigma_{\perp}(r)$ follows a $-0.92 \pm 0.04$ radial power law up to about 270 AU. Similar distributions are found for disks around T Tauri stars (e.g. Dutrey et al. 1996). The modeling of cloud core infall with no viscosity also predicts disk surface densities proportional to $r^{-1}$ (Lin \& Pringle 1990). Similarly, the surface density of the solar nebula during the disk dissipation is expected to follow a $r^{-1}$ radial power law under the assumption that the utilization of available solids to built planets in the Solar System is not efficient (Cameron 1995). In that frame, and given the conclusions on the optical thickness in Sect. 3.3.2, the inner part of the disk can rather be considered of first generation.

Outside $270 \mathrm{AU}, \sigma_{\perp}(r)$ falls as $r^{-3.5 \pm 0.2}$. A similar discontinuity of the dust distribution has already been reported in the case of the $\beta$ Pictoris disk. If the dynamics of the HD 100546 disk further than $270 \mathrm{AU}$ is similar to that of the outer part of $\beta$ Pictoris disk (namely, supplied in small grains by collisions among planetesimals and radiation pressure), we would expect the disk radial density distribution to follow a -4 radial power law (Lecavelier des Etangs et al. 1996). Such a disk, assumed optically thin and seen perfectly edge-on, indeed produces a midplane surface brightness proportional to $r^{-5}$ (Nakano 1990) as typically observed for $\beta$ Pictoris (Heap et al. 2000). The latter conclusion also requires quite isotropic scattering properties. With these assumptions, the HD 100546 disk would then flare as $r^{0.5 \pm 0.2}$. But the dynamical similarities between the HD 100546 and $\beta$ Pictoris disks at large distances remains an open issue.

\subsubsection{On the NE-SW brightness asymmetry}

What can produce the NE-SW brightness asymmetry if this is not an artifact due to instrumental effects? Although dust distribution asymmetries cannot be ruled out, the fact that the brightness asymmetry of the HD 100546 disk occurs in a direction close to its minor axis tends to indicate that this would be rather due to scattering properties.

In the case of an optically and a geometrically thin inclined disk, anisotropic scattering properties can produce similar asymmetries but they should occur whatever the distance from the star. In the present case, the asymmetry mostly appears at short distances where the normal optical thickness reaches values close to 0.1 .

The transition between the (marginally) optically thick regime in the inner disk and the optically thin regime at large distances combined with anisotropic scattering properties may explain the shape of the observed NE-SW brightness asymmetry. Further observations and models are needed to confirm this issue.

\subsubsection{Dust temperature and minimum grain size in the disk}

Hu et al. (1989) first claimed that the HD 100546 dust disk consists of a cold and a hot dust populations to account for the overall IR excesses. The relatively high spectral resolution of ISO spectra between $\lambda=2.4 \mu \mathrm{m}$ and $180 \mu \mathrm{m}$ lead Malfait et al. (1998) to propose temperature ranges for the two dust populations. Figure 6 shows these four isotherms as a function of the distance from the star and of the minimum grain size $a_{\text {min }}$ in the disk. The light-grey areas represent then the two dust populations. We only 


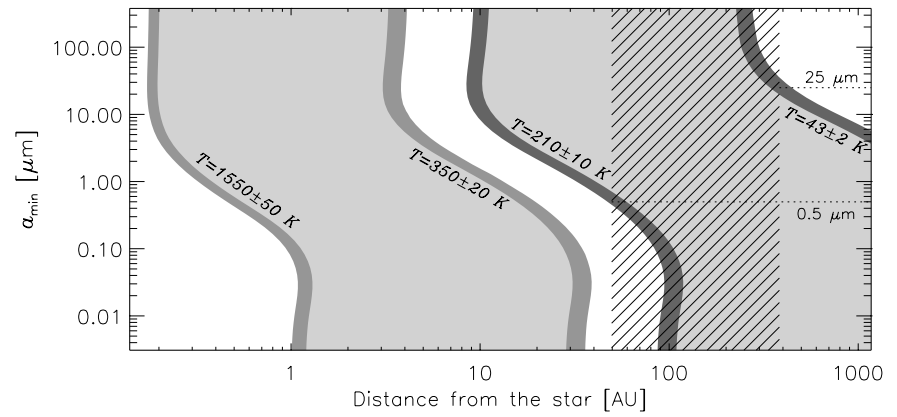

Fig. 6. Four mean isotherms of amorphous grains versus the distance from the star and the minimum grain size in the disk. The dark (resp. grey) isotherms correspond to the inner and outer boundaries of the cold (resp. hot) dust population proposed by Malfait et al. (1998) from ISO spectra. The dashed area indicates the radial range in which the disk is detected in scattered light

consider amorphous grains since amorphous silicates are supposed to be at least ten times more numerous than crystalline ones (Malfait et al. 1998).

In terms of spatial extent, the cooler population derived by Malfait et al. (1998) is consistent with the dust resolved in scattered light if the grains are larger than $\sim 0.5 \mu \mathrm{m}$ (see Fig. 6 : intersection of the light-grey and dashed areas). Indeed, grains at $T=210 \pm 10 \mathrm{~K}$ (the temperature corresponding to the inner edge of the colder population) that are smaller than a half micron lie further than $\sim 50 \mathrm{AU}$. Such grains would induce a detectable cut-off in the dust distribution somewhere between $50 \mathrm{AU}$ and $100 \mathrm{AU}$, which is not observed.

\subsubsection{Consistency of the scattered light image with IR excesses}

The dust resolved in scattered light, colder than $\sim 210 \mathrm{~K}$ according to Malfait et al. (1998), is expected to be responsible for most of the long wavelength IR excesses. At $100 \mu \mathrm{m}$, the SED is mainly featureless and the IR excess mostly comes from an underlying continuum (Malfait et al. 1998). We can then compare the observed flux at $100 \mu \mathrm{m}$ to the thermal emission implied by the normal surface densities deduced from present scattered light images in the optically thin approach (Fig. 4).

Figure 7 shows this comparison versus $a_{\min }$ for two different disk inner edges: the first one at $50 \mathrm{AU}$ and the second at $10 \mathrm{AU}$. In the latter case, $\sigma_{\perp}(r)$ has been extrapolated down to $10 \mathrm{AU}$ with a -0.92 radial power law. The distance of $10 \mathrm{AU}$ has been chosen because it roughly corresponds to the position of $210 \mathrm{~K}$ grains larger than the blow-out size limit ( $\sim 13 \mu \mathrm{m}$, Figs. A.1 and 6$)$.

Whatever $a_{\min }$, the predicted flux never reaches the observed limit. The optically thin approach does not then force any upper limit on the minimum grain size in the disk. Even grains larger than $10-20 \mu$ m lying in the $10 \mathrm{AU}-$ inner-edge disk radiate a flux 2.5 to 3 times smaller than the measured emission.

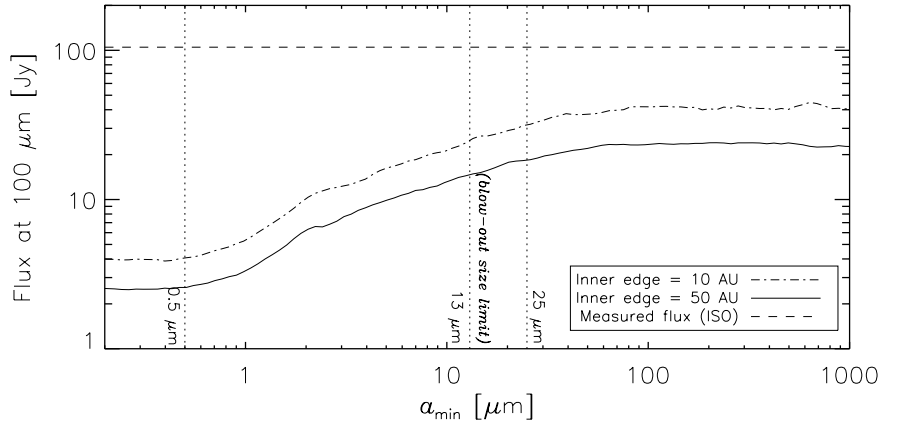

Fig. 7. Contribution to the $\lambda=100 \mu \mathrm{m}$ thermal emission of the dust population resolved in scattered light down to $50 \mathrm{AU}$ as a function of the minimum grain size $a_{\min }$ (plain line). The dotted-dashed line corresponds to the same population but extrapolated down to $10 \mathrm{AU}$. The uncertainties in this estimation are: $1 /$ the $\sim 10 \%$ uncertainty in the scattered image photometry, 2/ the uncertainty in the anisotropic scattering properties (we have assumed $f\left(90^{\circ}\right)=1 / 4 \pi$ for simplicity, see Appendix A) 3/ all the molecules detected with ISO which could partially change the mean grain albedo and 4 / the exact surface density if the optical depth is underestimated

As already noticed, the optically thin approach may underestimate the surface density, indicating that part of the dust mass does not contribute to scatter light images but may emit at $100 \mu \mathrm{m}$. For instance, with the assumed dust distribution, increasing the dust mass by a factor of 40 would still be compatible with the observations if the grains are submicronic.

\section{SAO 206462}

\subsection{The HD 135344 multiple system}

The F4Ve star SAO 206462 (Waelkens et al. 1990a,b) forms a visual binary system named HD 135344 with SAO $206463 \sim 20.4^{\prime \prime}$ away almost in the Northern direction. The latest star, that we used as the comparison star during our HST/NICMOS2 observations, has a spectral type between F2 (CDS) and A2 (Myers et al. 1998). Among our targets, SAO 206462 is the less luminous star but exhibits the largest relative infrared excess $\left(L_{\mathrm{IR}} / L_{*}=0.67\right.$, Coulson et al. 1995). Dust emission features at $3.29 \mu \mathrm{m}, 7.8 \mu \mathrm{m}$ and $11.3 \mu \mathrm{m}$ have been evidenced by Coulson et al. (1995) and attributed to aromatic molecules of a few angströms (Sylvester et al. 1996). CO is also detected by Zuckerman et al. (1995) and Coulson et al. (1998) whose abundance suggests a low gas to dust ratio $\left(810^{-3}\right.$, Coulson et al. 1998) if CO is representative of the gas content (i.e. not depleted with respect to the other species) consistent with that of Vega-like stars.

Coulson et al. 1995 and Malfait et al. (1998) derived from the full SED modeling a two dust component description of the disk. However, without imaging, the cooler dust that is present in the outer part of the disk ( $>$ typically $10 \mathrm{AU}$ ) is not precisely constrained. Moreover, as shown by Kenyon \& Hartmann (1997), a single thin (not flared) 


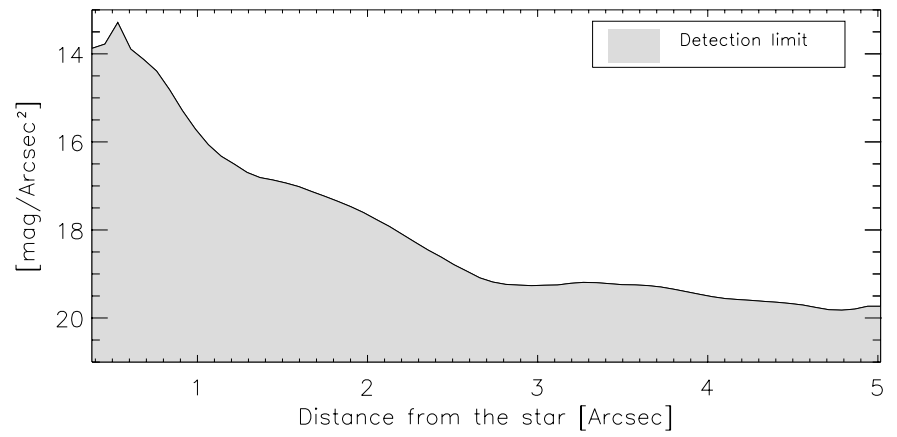

Fig. 8. SAO 206462 detection limit at $1.6 \mu \mathrm{m}$ with the HST/NICMOS2 camera

disk cannot account for the observed infrared excess and leads Sylvester et al. (1997) to propose either the presence of an envelope or accretion activity.

\subsection{Results}

\subsubsection{Disk detection limit}

No obvious feature that could be attributed to circumstellar dust is detected in the $1.6 \mu \mathrm{m}$ images of SAO 206462 (Fig. 10). The corresponding detection limit (Fig. 8) shows that at $1.6 \mu \mathrm{m}$ the disk is fainter than 13.5$14 \mathrm{mag} \operatorname{arcsec}^{-2}$ further than $0.4^{\prime \prime}(\sim 35 \mathrm{AU})$ and fainter than $16 \mathrm{mag} \mathrm{arcsec}^{-2}$ at $93 \mathrm{AU}\left(1.1^{\prime \prime}\right)$, i.e. close to the inner disk edge derived by Sylvester et al. (1997) from SED modeling. In the optically thin approach, this can be interpreted in terms of an upper limit on the normal optical thickness of about $10^{-2}$ (Fig. 9) outside of $0.4^{\prime \prime}$ in radius (35 AU). The dust material further than $35 \mathrm{AU}$ (if any) then is either optically thin at $1.6 \mu \mathrm{m}$ or does not contribute at this wavelength due to starlight occultation by the dust closer to the star.

\subsubsection{Implications for the dust distribution}

With the rough assumption that the dust surface density is proportional to $r^{-1.2}$ (as typically observed for young stars and which implies the larger mass), the dust mass between $35 \mathrm{AU}$ and $420 \mathrm{AU}$ would be less than: $2270 \times \sigma_{\perp, r=35 \mathrm{AU}}^{\text {upper limit }} M_{\oplus}$. It corresponds to $\sim 0.25 M_{\oplus}$ assuming $\sigma_{\perp, r=35 \mathrm{AU}}^{\text {upper limit }} \sim 10^{-4} \mathrm{~g} \mathrm{~cm}^{-2}$. This must be compared to the few Earth masses required to reproduce the full SED (Sylvester et al. 1997; Coulson et al. 1998) assuming moreover a maximum grain size at least 10 times smaller than for present work.

A large fraction of the dust mass (optically thin approach) or at least a large amount of dust (optically thick approach) is then certainly confined within the first few tens of AU. This is consistent with the mean cold population position of $23 \mathrm{AU}$ derived by Coulson et al. (1995) but not with Sylvester et al. (1997) (who assume very small grains in the disk, $\sim 50 \AA$ ). Present results agree with the
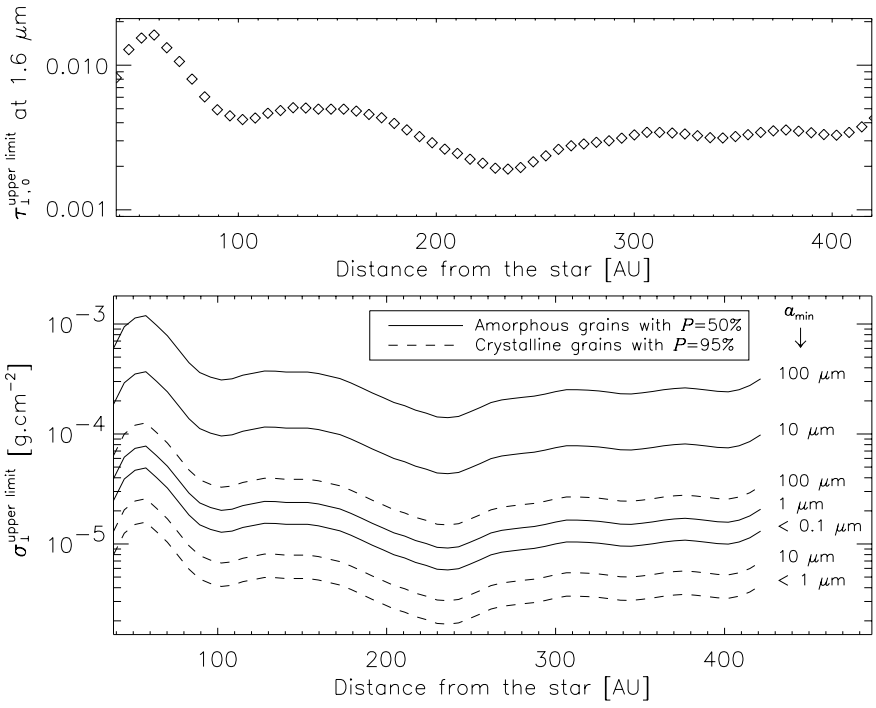

Fig. 9. Upper limits on the disk normal optical thickness at $\lambda=1.6 \mu \mathrm{m}$ (upper panel) and on the dust surface density as a function of the minimum grain size $a_{\min }$ (lower panel) for SAO 206462 assuming that the disk is optically thin

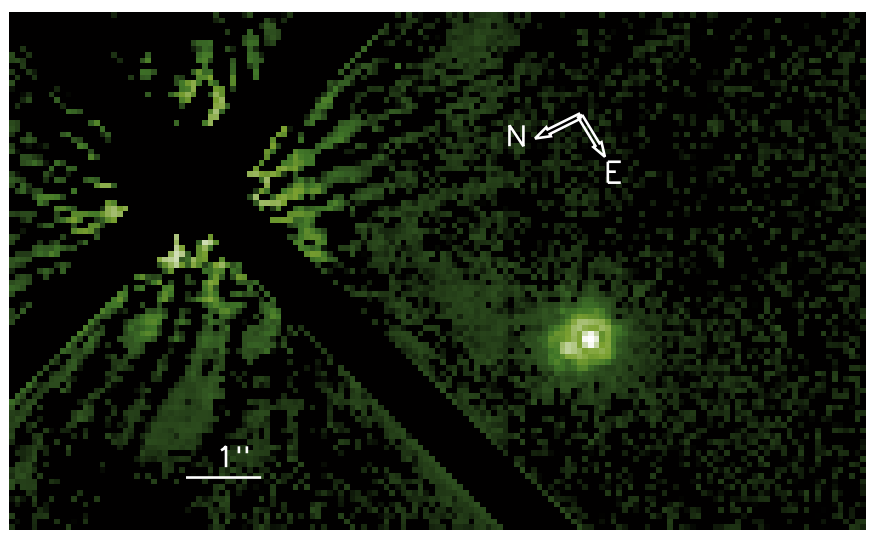

Fig. 10. The close binary system detected in the vicinity of SAO 206462 at $1.6 \mu \mathrm{m}$ with the HST/NICMOS2 camera

disk size limit of $\sim 60 \mathrm{AU}$ inferred by Jayawardhana et al. (2000) from their unresolved 10 and $18 \mu \mathrm{m}$ images.

\subsubsection{A tight binary system close to SAO 206462}

A close binary system in the SW direction is detected in the vicinity of SAO 206462 (Fig. 10). The brightest companion is $5.8^{\prime \prime} \pm 0.15^{\prime \prime}$ away from $\mathrm{SAO} 206462$ at $\mathrm{PA}=$ $129.8^{\circ} \pm 0.8^{\circ}$. The two close companion stars are separated by only $0.32^{\prime \prime} \pm 0.04^{\prime \prime}$ almost aligned along the NS axis; we find a PA of $352^{\circ} \pm 7$ for the third companion with respect to the brightest one. In Filter F160W, the secondary companion has a magnitude of $15.2 \pm 0.1$ and the third companion is about 6.3 times fainter $(\mathrm{mag}=17.2 \pm 0.1)$. 


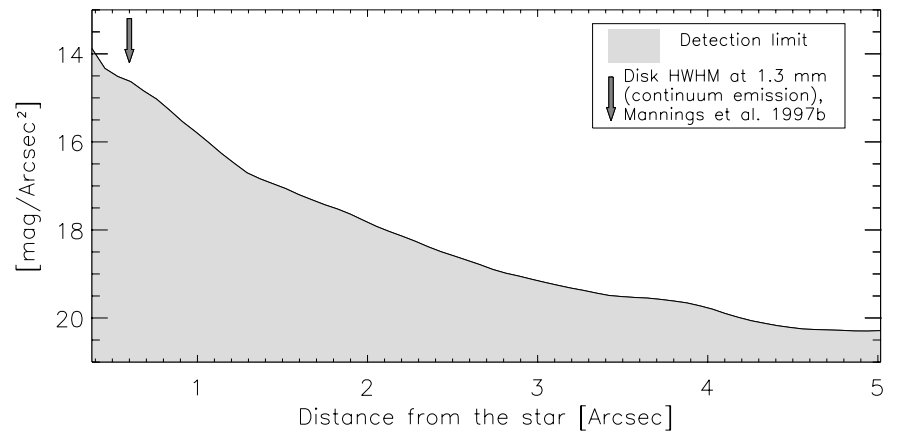

Fig. 11. MWC 480 detection limit at $1.6 \mu \mathrm{m}$ with the HST/NICMOS2 camera

\section{MWC 480}

\subsection{A sub-arcsec dust disk}

Among our sample, MWC 480 is the only one for which circumstellar gas has been imaged (Mannings et al. 1997b). The resolved structure shows Doppler shifts characteristic of the disk rotation over an angular scale of a few arcsec and inclined by $\sim 60^{\circ}$ from edge-on. Meanwhile, the dust continuum emission in the millimeter range also appears elongated in the same direction but is less extended $\left(H W H M \sim 0.6^{\prime \prime}\right.$ or $\left.\sim 80 \mathrm{AU}\right)$.

Two dust populations seem required to account for the overall infrared excesses. For instance, a combination of a geometrically thin disk and an envelope is proposed by Miroshnichenko et al. (1999) (see also Malfait et al. 1998). As in the case of $\beta$ Pictoris, the $10 \mu \mathrm{m}$ spectrum of MWC 480 shows features attributed to silicates (in particular crystalline olivine) similar to that of long-period comets in our solar system (e.g. comet Levy, Silko et al. 1999).

\subsection{Detection limit and implications for the dust distribution}

According to the detection by Mannings et al. (1997b), the dust disk is expected inside an angular region smaller than $1^{\prime \prime}$ in radius, a region where it is challenging to resolve faint structures because of such factors as PSF subtraction residues and diffraction spikes. Actually, very close to the edge of the mask, we do not find any convincing features which would clearly reveal the presence of the circumstellar disk. The azimuthally averaged noise in the image, interpreted in terms of detection limit, is shown in Fig. 11.

We applied in the case of MWC 480 strictly the same technique as the one used to derive some interesting properties of the SAO 206462 disk. Similarly, we conclude that either the disk further than $60 \mathrm{AU}$ is optically thin at $1.6 \mu \mathrm{m}$ or that the starlight is at least partially masked by a large amount of dust inside $60 \mathrm{AU}$ (Fig. 12).

For instance, if we constrain the dust mass between $60 \mathrm{AU}$ and $660 \mathrm{AU}$ to be smaller than $170 M_{\oplus}$ in solids and assuming $\sigma_{\perp} \propto r^{-1.75}$ (Mannings et al. 1997b), we
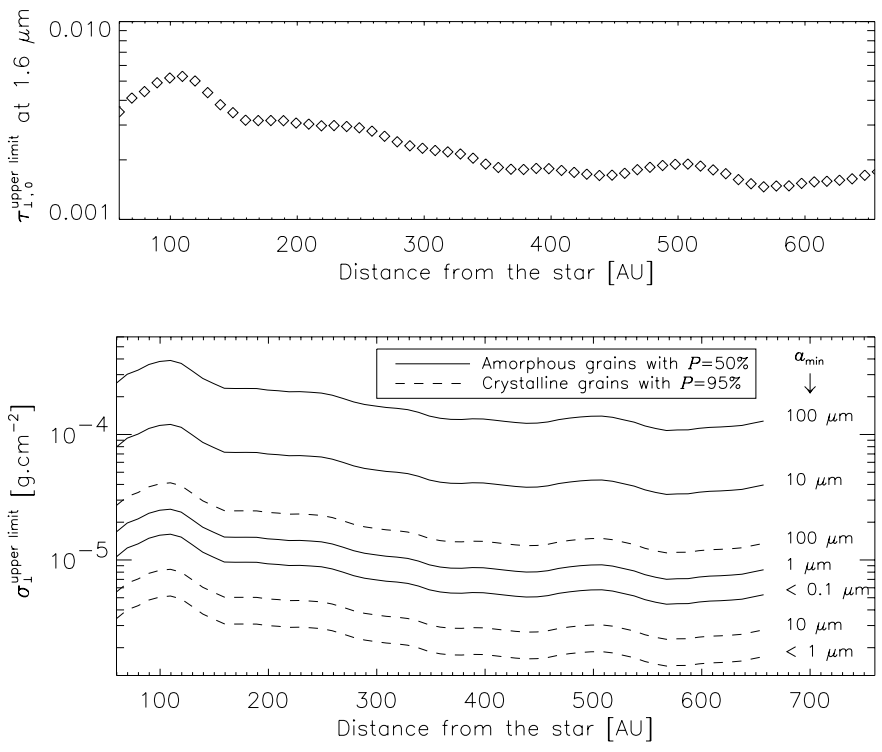

Fig. 12. Upper limits on the MWC 480 disk optical thickness at $\lambda=1.6 \mu \mathrm{m}$ and on the dust surface density as a function of the minimum grain size $a_{\mathrm{min}}$

find an upper limit on the surface density at $60 \mathrm{AU}$ of $610^{-2} \mathrm{~g} \mathrm{~cm}^{-2}$. This is consistent with the results in the optically thin approach from our scattered light observations (Fig. 12) even if grains are as large as a few hundred microns and even if we rather assume that only $10 \%$ of the dust mass lies further $60 \mathrm{AU}$. The present observations confirm then that most of the the dust mass is confined inside the first hundred AU, as shown by Mannings et al. (1997b).

\section{Conclusion}

Among our four HST/NICMOS2 Cycle 7 targets, two circumstellar disks have been resolved in scattered light. In this paper, we report the positive detection of an extended inclined disk around HD 100546 a typical transient oldPMS star expected to be in a disk dissipating phase. The shape of the derived surface density, the normal optical depth and, if confirmed, the brightness asymmetry along the minor axis of the inclined disk indeed indicate that the HD 100546 circumstellar environment certainly traces a stage between "first generation" massive disks around PMS stars and those of "second generation" around MS stars (Vega-like disks).

Disk non-detections are reported for both SAO 206462 and MWC 480. In each case, the detection limit in scattered light allows us to infer constraints on the dust location consistent with observations at other wavelengths.

Acknowledgements. We thank the referee, Dr. M. L. Sitko, for helpful suggestions. 

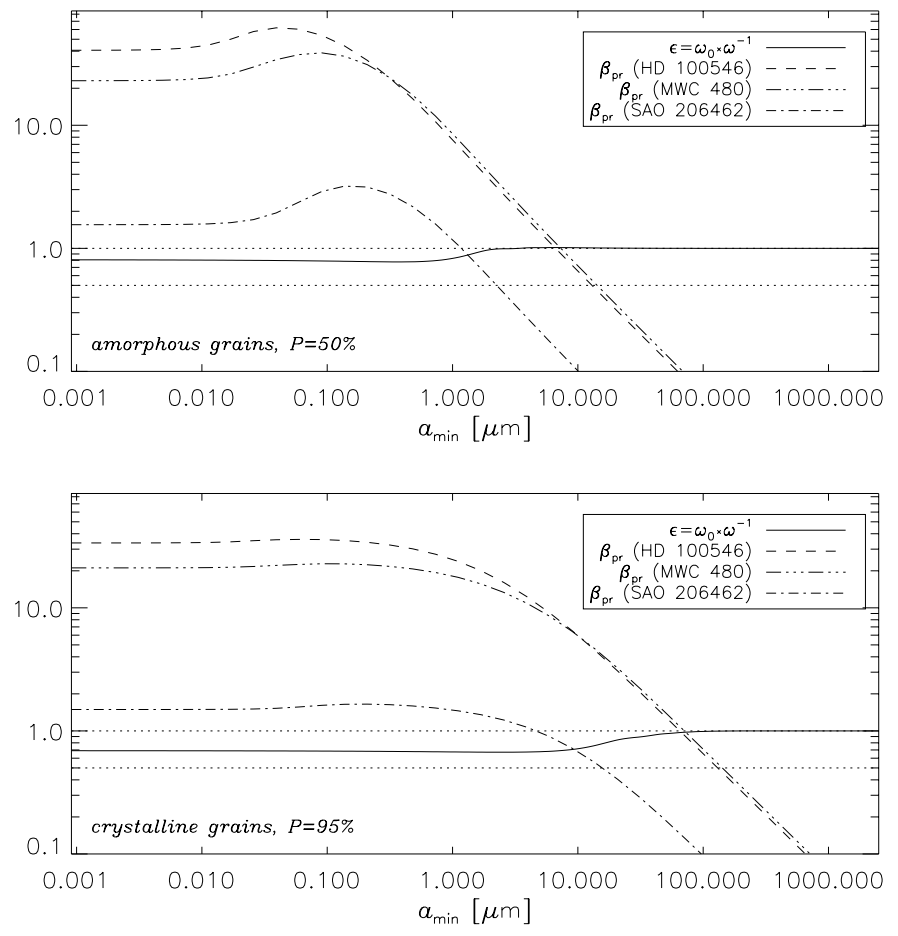

Fig. A.1. Correcting factor $\epsilon$ at $\lambda=1.6 \mu \mathrm{m}$ as a function of minimum grain size $a_{\text {min }}$ (see text). The ratios of radiation pressure to gravitational forces $\beta_{\mathrm{pr}}$ have been computed for grains with size $a_{\min }$ in the vicinity of HD 100546, MWC 480 and SAO 206462 (assumed to be a F4V star with $L_{*} \simeq 2.5 L_{\odot}$ and $M_{*} \simeq 1.2 M_{\odot}$, Allen 1973)

\section{Appendix A: Disk normal optical thickness}

The disk normal optical thickness can be straightforwardly derived from the normal surface brightness if we assume that the disk is axisymmetrical and optically thin.

More precisely, the disk normal surface brightness in scattered light is:

$S B_{\perp}(r) \simeq \Phi_{\lambda} r^{-2} f\left(90^{\circ}\right) \times\left\langle\sigma_{\text {sca }}\right\rangle_{a} n_{\perp}(r)$

where: $\Phi_{\lambda}$ is the total received flux $($ star+disk) at the considered wavelength $\lambda, r$ is the distance from the star, $f$ is the scattering phase function, $\left\langle\sigma_{\text {sca }}\right\rangle_{a}$ is the scattering cross-section averaged over the grain size distribution and $n_{\perp}(r)$ is the grain normal surface density distribution. This equation assumes that grain properties do not strongly depend on $r$.

On the other hand, the normal optical thickness is given by:

$$
\begin{array}{r}
\tau_{\perp}(r)=\left\langle\sigma_{\text {ext }}\right\rangle_{a} n_{\perp}(r)=\omega^{-1} \times\left\langle\sigma_{\text {sca }}\right\rangle_{a} n_{\perp}(r) \\
\text { with: } \omega=\frac{\left\langle\sigma_{\text {sca }}\right\rangle_{a}}{\left\langle\sigma_{\text {ext }}\right\rangle_{a}}
\end{array}
$$

where $\left\langle\sigma_{\text {ext }}\right\rangle_{a}$ is the averaged extinction cross-section. Finally, we have:

$\tau_{\perp}(r) \simeq \omega^{-1} \Phi_{\lambda}^{-1} f\left(90^{\circ}\right)^{-1} r^{2} S B_{\perp}(r)$.

The $\omega$ ratio depends on the wavelength, on the grain size distribution and on grain properties (shape, chemical

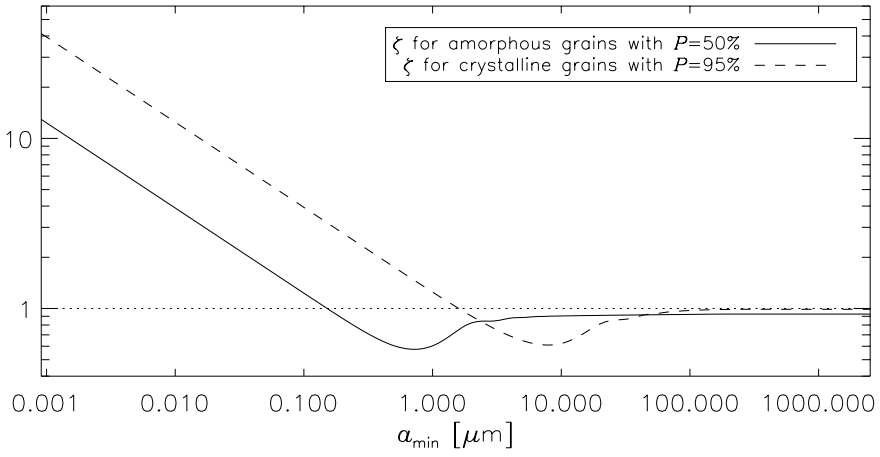

Fig. B.1. Correcting factor $\zeta$ at $\lambda=1.6 \mu \mathrm{m}$ as a function of minimum grain size $a_{\text {min }}$ (see text)

composition...). In the following, we assume $\lambda=1.6 \mu \mathrm{m}$ and a collisional grain size distribution proportional to $a^{-3.5}$ between $a_{\min }$ and $a_{\max }$. Grains are assumed to be spherical ( $a$ is the radius) and $a_{\max }$ is fixed to $1 \mathrm{~cm}$. We adopt the two types of grains proposed in Augereau et al. (1999a), namely: amorphous "ISM-like grains" with porosity $P$ of about $50 \%$ and crystalline "comet-like grains" with large porosity $(P \sim 95 \%)$. Grains are assumed to be made of silicates, organic refractories and a small amount of water ice (10\%). We use the Maxwell-Garnett effective medium theory to compute the complex index of refraction of the aggregate and the Mie theory to derive the optical properties.

For $a_{\min } \gg \lambda$, the $\omega$ ratio reaches a constant value $\omega_{0}$ close to 0.5 (Bohren \& Huffman 1983). Then if we note:

$$
\begin{array}{r}
\tau_{\perp, 0}(r)=2 \Phi_{\lambda}^{-1} f\left(90^{\circ}\right)^{-1} r^{2} S B_{\perp}(r) \\
\text { and } \epsilon=\omega_{0} \times \omega^{-1}
\end{array}
$$

we have:

$\tau_{\perp}(r) \simeq \epsilon \tau_{\perp, 0}(r)$

where $\epsilon$ represents a correcting factor which must be applied for the smallest $a_{\mathrm{min}}$, where the geometric optics approximation does not apply. $\epsilon$ versus $a_{\min }$ is shown in Fig. A.1 for the two types of grains. Note that for $a_{\text {min }}$ larger than a few $\frac{\lambda}{2 \pi(1-P)^{1 / 3}}, \omega$ is very close to the albedo of the smallest grains. For simplicity, we assume that grains scatter isotropically, then: $f\left(90^{\circ}\right) \simeq 1 / 4 \pi$.

\section{Appendix B: Disk normal surface density}

The normal surface density of the disk is obtained by:

$\sigma_{\perp}(r)=\left\langle\frac{4}{3} \pi \rho a^{3}\right\rangle_{a} \times n_{\perp}(r) \simeq \zeta \sigma_{\perp, 0}(r)$

with: $\sigma_{\perp, 0}(r)=\frac{2}{3} \rho_{\mathrm{g}} \tau_{\perp, 0}(r) \sqrt{a_{\min } a_{\max }}$

$$
\text { and: } \zeta=\frac{\left\langle\pi a^{2}\right\rangle_{a}}{\left\langle\sigma_{\mathrm{sca}}\right\rangle_{a}},
$$

assuming $\omega_{0} \simeq 0.5$. The grain density $\rho_{\mathrm{g}}$ depends on the grain properties. It is about $1.2 \mathrm{~g} \mathrm{~cm}^{-3}$ for amorphous 
grains and ten times smaller for crystalline grains due to the large porosity.

The $\zeta$ ratio plotted in Fig. B.1 is the correcting factor (as $\epsilon$ for the normal optical thickness). It must be applied for the smallest $a_{\mathrm{min}}$, i.e. where the more numerous grains (those with sizes close to $a_{\min }$ ) are not efficient scatterers. But in the same time (for the same smallest $a_{\min }$ ), $\zeta$ is proportional to $1 / \sqrt{a_{\min }}$ and then the normal surface density does not depend any more on the minimum grain size.

The normal surface density $\sigma_{\perp}(r)$ can therefore be inferred from the observed surface brightness if the disk is seen almost pole-on. If the disk is not resolved, it is also possible to derive an upper limit on $\sigma_{\perp}(r)$ from the detection limit. Obviously, the main uncertainty on $\sigma_{\perp}(r)$ comes from the lack of strong constraints on the minimum and maximum grain sizes in the disk.

\section{References}

Allen, C. W. 1973, Astrophys. Quant., Third edition (The Athlone Press LTD)

Augereau, J. C., Lagrange, A. M., Mouillet, D., Papaloizou, J. C. B., \& Grorod, P. A. 1999a, A\&A, 348, 557

Augereau, J. C., Lagrange, A. M., Mouillet, D., \& Ménard, F. 1999b, A\&AL, 350, 51

Aumann, H. H., Gillett, F. C., Beichman, C. A., et al. 1984, ApJL, 278, 23

Backman, D., \& Paresce, F. 1993, in Protostars and Planets III, ed. E. H. Levy, \& Lunine (Tucson: University of Arizona Press) 1253

Barrado y Navascués, D., Stauffer, J. R., Song, I., \& Caillault, J.-P. 1999, ApJL, 520, 123

Beust, H., Karmann, C., \& Lagrange, A. M. 2000, A\&A, submitted

Bohren, C. F., \& Huffman, D. R. 1983, Absorption and scattering of light by small particles (Wiley, New-York)

Bouwman, J., et al. 2000, IAU Symp. S202 Manchester, UK

Cameron, A. G. W. 1995, Meteoritics, 30, 133

Charbonneau, D. B., Brown, T. M., Latham, D. W., \& Mayor, M. 2000, ApJL, 529, 45

Clarke, D., Smith, R. A., \& Yudin, R. V. 1999, A\&A, 347, 590

Coulson, I. M., \& Walther, D. M. 1995, MNRAS, 274, 977

Coulson, I. M., Walther, D. M., \& Dent, W. R. F. 1998, MNRAS, 296, 934

Crovisier, J., Leech, K., Bockelée-Morvan, D., et al. 1997, Sci, 275,1904

de Winter, D., Grady, C. A., van den Ancker, M. E., Pérez, M. R., \& Eiroa, C. 1999, A\&A, 343, 137

Dunkin, S. K., Barlow, M. J., \& Ryan, S. G. 1997, MNRAS, 286,604

Dutrey, A., Guilloteau, S., Duvert, G., et al. 1996, A\&A, 309, 493

Grady, C. A., Perez, M. R., Talavera, A., et al. 1996, A\&AS, 120,157

Grady, C. A., Sitko, M. L., Bjorkman, K. S., et al. 1997, ApJ, 483,449

Greaves, J. S., Holland, W. S., Moriarty-Schieven, G., et al. 1998, ApJL, 506, 133

Habing, H. J., Dominik, C., Jourdain de Muizon, M., et al. 1999, Nat, 401, 456

Heap, S. R., Lindler, D. J., Lanz, T. M., et al. 2000, ApJ, 530
Henning, Th., Launhardt, R., Steinacker, J., \& Thamm, E. 1994

Henning, Th., Burkert, A., Launhardt, R., Leinert, C., \& Stecklum, B. 1998, A\&A, 336, 565

Holland, W. S., Greaves, J. S., Zuckerman, B., et al. 1998, Nat, 392, 788

Hu, J. Y., Thé, P. S., \& de Winter, D. 1989, A\&A, 208, 213

Jayawardhana, R., Fisher, R. S., \& Telesco, C. M. 2000, Ph.D. Thesis

Kenyon, S. J., \& Hartmann, L. 1997, ApJ, 323, 714

Krist, J. E., Stapelfeldt, K., Ménard, F., Padgett, D., \& Burrows, C. 2000, ApJ, 538, 793

Lagrange, A. M., Backman, D., \& Artymowicz, P. 2000, in PPIV, 639-672

Lecavelier des Etangs, A., Vidal-Madjar, A., \& Ferlet, R. 1996, A\&A, 307, 542

Lin, D. N. C., \& Pringle, J. E. 1990, ApJ, 358, 515

Malfait, K., Waelkens, C., Waters, L. B. F. M., et al. 1998, A\&AL, 332, 25

Mannings, V., \& Sargent, A. I. 1997a, ApJ, 490, 792

Mannings, V., Koerner, D. W., \& Sargent, A. I. 1997b, Nat, 388,555

Miroshnichenko, A., Ivezić, Z., \& Elitzur, M. 1997, ApJL, 475, 41

Miroshnichenko, A., Ivezić, Z., Vinković, D., \& Elitzur, M. 1999, ApJL, 520, 115

Myers, J. R., Sande, C. B., Miller, A. C., Warren Jr., W. H., \& Tracewell, D. A. 1998, Sky2000 Master Catalog, Goddard Space Flight Center, Flight Dynamics Division

Nakano, T. 1990, ApJL, 355, 43

Padgett, D. L., Brandner, W., Stapelfeldt, K. R., et al. 1999, AJ, 117, 1490-1504

Pantin, E., Waelkens, C., \& Lagage, P. O. 2000, A\&AL, in press

Queloz, D., Mayor, M., Weber, L., et al. 2000, A\&A, 354, 99

Schultz, A. B., Storrs, A. D., \& Fraquelli, D., Instrument Science Report NICMOS-99-006

Sitko, M. L., Grady, C. A., Lynch, D. K., Russell, R. W., \& Hanner, M. S. 1999, ApJ, 510, 408

Sylvester, R. J., Skinner, C. J., Barlow, M. J., \& Mannings, V. 1996, MNRAS, 279, 915

Sylvester, R. J., Skinner, C. J., \& Barlow, M. J. 1997, MNRAS, 289, 831

van den Ancker, M. E., Thé, P. S., Tjin A Djie, H. R. E., et al. 1997, A\&A, 324, L33

van den Ancker, M. E., de Winter, D., \& Tjin A Djie, H. R. E. 1998, A\&A, 330, 145

Vieira, S. L. A., Pogodin, M. A., \& Franco, G. A. P. 1999, A\&A, 345, 559

Waelkens, C., Engelsman, E., Waters, L. B. F. M., Van der Veen, W. E. C. J., \& Trams, N. R. 1990a, in From Miras to Planetary Nebulae: Wich Path for Stellar Evolution?, ed. M. O. Menessier, \& A. Omont (Éditions Frontières, France) 470

Waelkens, C., Van Winckel, H., \& Trams, N. R. 1990b, in IAU Symp. 145, Evolution of stars: The Photospheric Abundance Connection (Poster Papers) 21, ed. Michaud, Tutukov, \& Bergevin (Montreal, Canada)

Waters, L. B., \& Waelkens, C. 1998, ARA\&A, 36, 233

Weinberger, A. J., Becklin, E. E., Schneider, G., et al. 1999, ApJL, 525, 53

Yudin, R. V., \& Evans, A. 1998, A\&AS, 131, 401

Zuckerman, B., \& Becklin, E. E. 1993, ApJ, 414, 793

Zuckerman, B., Forveille, T., \& Kastner, J. H. 1995, Nat, 373, 494 